\title{
Diagnostica metabolica della calcolosi renale. Casi clinici (Parte I)
}

\author{
Corrado Vitale ${ }^{1}$, Amelia Rodofili ${ }^{1}$, Francesca Bermond ${ }^{1}$, Alberto Tricerri ${ }^{1}$, Martino Marangella ${ }^{2}$ \\ ${ }^{1}$ SC Nefrologia e Dialisi, AO Ordine Mauriziano di Torino, Torino \\ ${ }^{2}$ Fondazione Scientifica Mauriziana ONLUS, Torino
}

\begin{abstract}
Metabolic diagnosis of nephrolithiasis. Clinical cases (Part I)
Nephrolithiasis is one of the most frequent causes of hospitalization in nephrology and urology units in our country and is an important source of discomfort in affected patients. Despite the availability of modern, minimally invasive endourological procedures to remove stones from the urinary tract, prevention of stone recurrences remains an essential strategy. Chemical analysis of stones, whether passed spontaneously or removed surgically, is a crucial step in etiological diagnostics aimed at devising adequate prevention strategies. Modern endoscopic lithotripsy techniques provide complete fragmentation of stones and, compared to extracorporeal shock wave lithotripsy, have the advantage of avoiding painful elimination of fragments through the urinary tract. This means, however, that stone fragments are often unavailable for analysis, thereby depriving the nephrologist of an important tool for diagnosis. As a consequence, metabolic evaluation tends to be the only means of establishing the etiology of stones and preventing their recurrence. Among patients attending our kidney stone center, we selected five clinical cases representative of a rational diagnostic workup in specific clinical contexts. Two of these nephrolithiasis cases (in a patient with osteoporosis and in a patient with primary hyperparathyroidism) are reported here (Part I); the remaining three will be discussed in the next issue of this journal (Part II).
\end{abstract}

Keywords: Cystinuria, Hyperoxaluria, Nephrolithiasis, Primary hyperparathyroidism, Urolithiasis

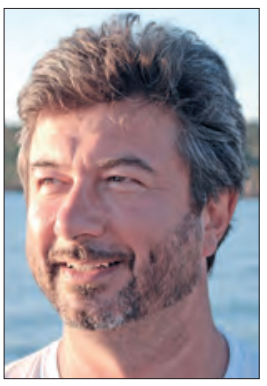

Corrado Vitale

\section{Introduzione}

La calcolosi renale è una malattia molto comune. Nei paesi industrializzati, è stata riportata una prevalenza nella popolazione generale variabile dall' $1 \%$ al $10 \%$, con punte fino al $20 \%(1)$. In Italia disponiamo di pochi dati aggiornati. Statistiche ISTAT ormai datate riportavano una prevalenza in crescendo dall'1.17\% all'1.84\% tra il 1983 e il 1993.

Accepted: September 26, 2016

Published online: October 19, 2016

Indirizzo per la corrispondenza:

Dr. Corrado Vitale

SC di Nefrologia e Dialisi

AO Ordine Mauriziano

Largo Turati 62

10128 Torino

covitale@mauriziano.it
Si tratta, tuttavia, di dati sottostimati, che si riferivano soltanto alle forme più gravi necessitanti di un ricovero ospedaliero.

Studi su scala limitata, ma più recenti, hanno stimato una prevalenza della calcolosi urinaria in Italia variabile tra il $6 \%$ e il $9 \%$, con un'incidenza di circa 100.000 nuovi casi/ anno $(2,3)$.

La nefrolitiasi è causa di danno biologico e di costi economici significativi, anche indiretti (perdita di giornate lavorative, di produttività, ecc.). Insieme all'insufficienza renale cronica e all'ipertrofia prostatica benigna, è una delle più frequenti cause di ricovero ospedaliero per motivi nefro-urologici.

Benché le moderne tecnologie abbiano significativamente modificato l'approccio urologico alla calcolosi renale, consentendo la rimozione incruenta di calcoli anche di cospicue dimensioni, la prevenzione delle recidive litiasiche resta a tutt'oggi irrinunciabile.

Tradizionalmente, la profilassi delle recidive di malattia iniziava con l'analisi della composizione chimica del calcolo urinario, espulso spontaneamente o rimosso chirurgicamente. Lo studio della biochimica sierica e urinaria costituiva, poi, il necessario approfondimento diagnostico, 
giacché calcoli della medesima composizione possono essere l'effetto di alterazioni metaboliche del tutto differenti tra loro (4).

Nel caso delle forme di calcolosi più complesse, quando non sia possibile l'espulsione spontanea dei calcoli, le moderne tecnologie consentono di ottenere la completa dissoluzione dei calcoli urinari con procedure endourologiche laser-assistite. Rispetto alle tecniche extracorporee (ESWL), più usate in un recente passato, la litotrissia endoscopica solleva il paziente dal disagio legato all'espulsione spontanea di frammenti residui. Tuttavia, proprio questa assenza di frammenti litiasici da analizzare priva il nefrologo di un prezioso momento diagnostico. Per questo motivo, oggi lo studio metabolico è spesso l'unico strumento disponibile per studiare l'eziologia della calcolosi renale e impostare un'efficace profilassi delle recidive.

Sono riportati qui cinque casi clinici illustrativi di un percorso ragionato di diagnostica metabolica della calcolosi renale in differenti contesti clinici: osteoporosi, iperparatiroidismo primitivo, iperuricemia secondaria, cistinuria e iperossaluria primitiva. La stretta collaborazione tra Nefrologo e Urologo è indispensabile per ottimizzare il percorso diagnostico e terapeutico della calcolosi renale.

\section{Caso clinico 1}

\section{Anamnesi}

Paziente nata nel 1951. Due gravidanze regolari (1978, 1983), menopausa nel 2005. Nel 2001, riscontro di ipertensione arteriosa e ipercolesterolemia, con prescrizione di Enalapril e Simvastatina.

Nel settembre del 2003, riscontro occasionale di ipercalcemia e diagnosi di iperparatiroidismo primitivo (PTH $184 \mathrm{pg} / \mathrm{mL}$ ). Consensualmente, vi era l'evidenza di nefrolitiasi radio-opaca (calcolo polare superiore sinistro di $7 \mathrm{~mm}$ e mesorenale destro di $4 \mathrm{~mm}$ ) e osteoporosi (MOC lombare: T score -3.13, Z score -2.27).

Nel gennaio del 2004, exeresi di adenoma paratiroideo basale destro, con normalizzazione dei livelli di calcemia e PTH.

Nel 2005, a causa di cefalee ricorrenti, fu eseguita una RMN encefalica che dimostrò esiti di pregressi eventi microischemici cerebrali.

All'epoca della prima visita nefrologica (2006), la paziente si presentava in ottime condizioni generali, svolgeva una vita molto attiva e praticava sport (sci, bicicletta). Non aveva mai manifestato coliche renali né era andata incontro a fratture. I valori pressori erano ottimamente controllati dalla terapia. In seguito alla diagnosi di calcolosi renale, la paziente aveva quasi eliminato dalla propria alimentazione il latte e i suoi derivati, che già in precedenza assumeva in modeste quantità. L'introduzione media di liquidi era di circa 1.5 litri al giorno.
Siero: urea, creatinina, acido urico, sodio, potassio, calcio totale e ionizzato, fosforo, cloro, magnesio, $\mathrm{pH}$, bicarbonato, PTH, $25(\mathrm{OH})$ Vitamina $D_{3}$, fosfatasi alcalina

Urine delle 24 ore: urea, creatinina, acido urico, sodio, potassio, calcio, fosforo, cloro, magnesio, ossalato, citrato, solfato, $\mathrm{pH}$, bicarbonato, ammonio, acidità titolabile. Test di Brand.

Urine a digiuno: calcio, citrato, creatinina, desossipiridinolina.

Fig. 1 - Screening metabolico per calcolosi renale. Indagini bioumorali sieriche e urinarie.

\section{Indagini biochimiche e strumentali}

Come primo inquadramento, furono prescritti: ecografia addominale, mineralometria ossea, Rx del rachide e indagini bioumorali per lo studio del metabolismo minerale e dello stato di saturazione urinaria per i sali litogeni. Queste ultime furono effettuate secondo il nostro protocollo più ampio (Fig. 1), dedicato in particolare alle nefrolitiasi altamente recidivanti e a quelle insorte in età giovanile oppure in contesti clinici ad aumentato rischio per disturbi del metabolismo osseo (p. es., età perimenopausale, osteopenia, osteoporosi, disendocrinie, sindromi da malassorbimento).

In base ai valori dei parametri urinari considerati nel protocollo, con un apposito software (Lithorisk ${ }^{\circ}$ ) viene calcolato lo stato di saturazione rispetto a ossalato di calcio ( $\mathrm{BCaOx}$ ), fosfato di calcio (ßbsh) e acido urico (BAU): valori di $\beta>1$ indicano sovrasaturazione, con rischio di precipitazione dei rispettivi sali litogeni.

Le urine costituiscono una soluzione concentrata ed è possibile riscontrare condizioni di sovrasaturazione anche in individui sani. Nella nostra esperienza, valori di $\mathrm{BCaOx}$ intorno a 4 e di ßbsh intorno a 1.5 sono di frequente riscontro anche in soggetti non affetti da nefrolitiasi calcica, mentre valori di $\beta A U>1.5$ sono osservabili soltanto eccezionalmente in pazienti non affetti da litiasi urica (5).

Nel protocollo riportato nella Figura 1, la fosfatasi alcalina (ALP) figura quale indice di bone formation e la desossipiridinolina urinaria (DPD) quale indice di bone resorption. II test di Brand è un indicatore semiquantitativo di livelli sopranormali di Cistina nelle urine, che, in caso di positività, devono essere confermati mediante il dosaggio diretto della Cistina urinaria.

I risultati più significativi delle rilevazioni di laboratorio e della densitometria ossea a cui fu sottoposta la paziente, sia delle prime del 2006 sia di quelle del successivo follow-up nefrologico, sono riportati nella Tabella I e discussi nel paragrafo seguente.

L'ecotomografia renale esibita nel corso della prima visita del 2006 dimostrò un quadro di nefrolitiasi sinistra di 7-8 mm e destra di $5 \mathrm{~mm}$, sostanzialmente stabile rispetto al dato di tre anni prima. 
La Rx della colonna vertebrale dimostrò una diffusa riduzione del tono calcico vertebrale, senza segni di frattura o deformità ossee.

\section{Diagnosi}

Il primo referto nefrologico della paziente riportava: "Nefrolitiasi calcica bilaterale, pregresso iperparatiroidismo primitivo, grave osteoporosi non complicata da fratture e severa deplezione di vitamina $D$, in una paziente con ipertensione arteriosa e microvasculopatia cerebrale".

Le indagini bioumorali basali (Tab. I, 2/2006) dimostrarono una funzione renale normale, senza recidive di iperparatiroidismo. Ridotti valori di calciuria, sia su urine di 24 ore che a digiuno, sono di comune riscontro nelle condizioni di insufficiente apporto nutrizionale di calcio; in questo caso, ciò era verosimilmente conseguente sia alla scarsa introduzione alimentare sia a un ridotto assorbimento intestinale, secondario alla grave carenza di Vitamina $\mathrm{D}$.

Complessivamente, il rischio litogeno urinario ( $\mathrm{BCaOx}$ e ßbsh) risultava assai contenuto, suggerendo che la condizione responsabile della litogenesi fosse stata la pregressa disendocrinia paratiroidea (6).

La densità minerale ossea dimostrò un quadro di osteoporosi conclamata, con valori di T score in ulteriore riduzione rispetto al 2003.

\section{Terapia}

Alla paziente furono prescritti una terapia idropinica e un'alimentazione normocalcica e ipo-ossalica, moderatamente iposodica, con un apporto di proteine animali limitato a una porzione al giorno, con supplementazione di Vitamina D (Didrogyl 5 gocce al giorno).

Questo caso clinico ha diverse peculiarità, che meritano alcuni commenti.

Il grave stato carenziale di Vitamina D e l'alimentazione ipocalcica rappresentano fattori di rischio aggiuntivi per lo sviluppo di una severa deplezione minerale ossea, di per sé già favorita dalla pregressa disendocrinia paratiroidea e dalla menopausa. Queste problematiche nutrizionali sono, oggi, di comune osservazione, giacché molti pazienti sono in sovrappeso e/o dislipidemici e sono invitati a limitare l'assunzione di formaggi. Inoltre, è, purtroppo, ancora diffusa la convinzione che una restrizione del calcio alimentare riduca il rischio di calcolosi renale, malgrado consolidate evidenze epidemiologiche dimostrino il contrario (7). Infine, l'esposizione diretta al sole (fonte primaria di Vitamina $\mathrm{D}$ ) è, oggi, perlopiù modesta, sia per i mutati stili di vita rispetto al passato sia per il diffuso impiego di creme protettive che attenuano molto gli effetti dei raggi solari.

Per i pazienti litiasici con deplezione minerale ossea, uno dei principali obiettivi terapeutici è quello di fornire un apporto

TABELLA I - Caso clinico 1. Dati metabolici e di densità minerale ossea

\begin{tabular}{|c|c|c|c|c|c|}
\hline & Val. normali & $2 / 2006$ & $5 / 2008$ & $3 / 2012$ & $6 / 2015$ \\
\hline Creatininemia (mg/dL) & $0.5-1.3$ & 0.7 & 0.7 & 0.8 & 0.8 \\
\hline Clear. Creatinina $(\mathrm{mL} / \mathrm{min})$ & $70-140$ & 85 & 90 & 78 & 81 \\
\hline Calcemia totale $(\mathrm{mg} / \mathrm{dL})$ & $8.2-10.2$ & 8.7 & 9.4 & 9.2 & 8.8 \\
\hline Fosforemia $(m g / d L)$ & $2.5-4.5$ & 3.4 & 4.0 & 4.0 & 4.1 \\
\hline PTH intatto $(p g / m L)$ & $11-70$ & 57.2 & 49.7 & 38.2 & 47.2 \\
\hline $250 \mathrm{H}$ Vitamina $\mathrm{D}_{3}(\mathrm{ng} / \mathrm{mL})$ & $30-80$ & 6.9 & 32.5 & 88.4 & 34.5 \\
\hline $\operatorname{ALP}(U / L)$ & $70-270$ & 230 & 263 & 205 & 276 \\
\hline Fosfaturia (mmol/24h) & $13-52$ & 23.1 & 19.7 & 22.5 & 18.5 \\
\hline Calciuria (mmol/24h) & $2.5-9$ & 1.9 & 3 & 2.75 & 2.9 \\
\hline Calciuria $(\mathrm{mg} / \mathrm{kg})$ & $<4$ & 1.3 & 2.1 & 1.9 & 2.1 \\
\hline Ossaluria (mmol/24h) & $<0.55$ & 0.36 & 0.28 & 0.31 & 0.30 \\
\hline Citraturia (mmol/24h) & $1.5-6.5$ & 2.3 & 2.9 & 3.1 & 3.2 \\
\hline Calcio/Creat. fasting ( $\mathrm{mg} / \mathrm{mg})$ & $<0.11$ & 0.07 & 0.10 & 0.10 & 0.13 \\
\hline DPD (nmol/mmol creat) & $2.3-7.4$ & 7.1 & 8.7 & 6.5 & 10.6 \\
\hline B CaOx & Satur. $=1$ & 3.5 & 2.6 & 3.0 & 2.7 \\
\hline ß bsh & Satur. $=1$ & 1.8 & 1.1 & 1.5 & 1.4 \\
\hline MOC lombare, T score & & -4.8 & -4.7 & -4.6 & -4.2 \\
\hline MOC lombare, Z score & & -3.8 & -3.5 & -3.1 & -2.5 \\
\hline
\end{tabular}


di calcio adeguato alle necessità scheletriche, in condizioni di sicurezza rispetto al rischio litogeno urinario.

Le Linee Guida della Società Italiana di Osteoporosi, Metabolismo Minerale e Malattie dello Scheletro (SIOMMMS) riportano, per donne in età menopausale che non siano in terapia ormonale sostitutiva, un fabbisogno di calcio di $1500 \mathrm{mg} / \mathrm{die}$, indicando come preferibile la supplementazione con gli alimenti rispetto a quella con compresse $(8,9)$. In questo contesto, un ruolo particolare può essere rivestito dalle acque minerali bicarbonato-calciche, soprattutto quelle con un contenuto di calcio intorno a $300-400 \mathrm{mg}$ per litro (10).

In pazienti con calcolosi renale, le acque bicarbonato calciche si sono dimostrate efficaci nel ridurre la sovrasaturazione urinaria per i sali litogeni; le consensuali riduzioni dei livelli sierici di PTH e dei marker di riassorbimento osseo suggeriscono una positivizzazione del bilancio esterno del calcio (11). Gli effetti positivi dell'assunzione di questo tipo di acque sul metabolismo osseo e l'equilibrio acidobase sono, in seguito, stati confermati anche in pazienti uremici (12).

Rispetto ai prodotti caseari, le acque bicarbonato-calciche non hanno effetti calciuretici aggiuntivi, essendo prive di proteine animali e contenendo solo minime quantità di sodio, mentre apportano significative quantità di valenze alcaline, che aumentano l'escrezione di citrato $(11,13)$ e hanno effetti favorevoli sul bilancio minerale (14).

Nel caso della nostra paziente, l'apporto dietetico raccomandato di calcio fu ottenuto mediante un regime alimentare che prevedeva limitate quantità di formaggi magri (apporto calcico complessivo stimato intorno a $600 \mathrm{mg} / \mathrm{die}$ ), integrato con due litri di acqua bicarbonato-calcica (apporto calcico additivo intorno ai $750 \mathrm{mg} /$ die).

Per quanto riguarda l'ossalato, questo è essenzialmente un end-product del metabolismo intermedio, sicché la quota di ossalato urinario ascrivibile alla sua assunzione in quanto tale, perlopiù tramite i vegetali, non supera generalmente il $10 \%$. Un'alimentazione molto ristretta per quanto riguarda i vegetali, oltre ad avere modesti effetti sull'ossaluria, implicherebbe uno sbilanciamento dietetico verso le proteine animali, fortemente prolitogene (15).

Nei nostri pazienti, riteniamo conveniente disporre una severa restrizione soltanto nei confronti dei cibi più ricchi di ossalato, come, per esempio: cacao, cioccolato, spinaci, bietole-costine, frutta secca, pomodori secchi e tè concentrato. Tra le verdure, consigliamo di prediligere quelle senza larghe foglie verdi, mentre non poniamo restrizioni sull'assunzione di frutta fresca.

In questa paziente, l'assenza di eventi fratturativi, la valida attività fisica e l'ampio spazio terapeutico nutrizionale indussero, in prima istanza, a soprassedere alla somministrazione di bisfosfonati o raloxifene (quest'ultimo, peraltro, controindicato in considerazione della vasculopatia cerebrale).

\section{Decorso clinico}

La paziente si sottopose a visite nefrologiche con cadenza annuale; i riscontri biochimici più significativi sono riportati nella Tabella I.

Nel maggio del 2008, fu riscontrabile un lieve e del tutto accettabile incremento della calciuria rispetto ai valori basali (comunque in limiti fisiologici), compatibile con il raddoppio dell'intake di calcio e il ripristino di normali livelli di Vitamina D. La lieve riduzione dell'ossaluria rispetto al controllo precedente è verosimilmente spiegabile con un minor assorbimento intestinale di ossalato, dovuto all'effetto chelante del calcio alimentare, mentre l'aumento della citraturia è conseguente al carico alcalinizzante delle acque bicarbonatocalciche (11).

$\mathrm{Nel} 2008$, i valori di densità minerale ossea confermarono una severa osteoporosi (T score: -4.7). Tuttavia, la sostanziale stabilità del dato densitometrico rispetto al 2006 costituiva una positiva inversione di tendenza, considerando il decorso ingravescente riscontrato tra il 2003 (T score $=-3.1$ ) e il 2006 ( T score $=-4.8$ ). A fronte di un apporto di calcio e Vitamina $D$ stabilmente corretto, indispensabile presupposto per un'efficace terapia con bisfosfonati, fu, quindi, prescritta una terapia con Alendronato $70 \mathrm{mg} / \mathrm{settimana}$.

Al controllo del marzo del 2012, la paziente era in ottime condizioni cliniche, senza recidive litiasiche. I valori di densità minerale ossea (T score) mostravano un minimo incremento rispetto a quelli del 2008 e del 2006. Ciò fu ritenuto comunque un segno di efficacia terapeutica, considerando che, in età menopausale, è fisiologico osservare una riduzione del T score. Tuttavia, dovendo la paziente essere sottoposta a interventi di implantologia dentaria, la terapia con bisfosfonati fu sospesa.

Il quadro ecografico del 2012 era del tutto sovrapponibile a quello del 2006 e il profilo metabolico non presentava elementi patologici. In considerazione dei livelli medio-alti di $25(\mathrm{OH})$ Vitamina $\mathrm{D}$, la somministrazione quotidiana di Calcifediolo fu sostituita con una terapia settimanale a base di Colecalciferolo (6.250 U).

Nel giugno del 2015, i valori di DPD, ALP e calciuria a digiuno risultarono aumentati rispetto ai controlli precedenti, suggestivi di una riattivazione del turnover osseo conseguente al venir meno dell'effetto inibitorio dei bisfosfonati. Dal momento che la densità minerale ossea mostrava progressivi miglioramenti ( score $=-4.2$ ) e la paziente rimaneva asintomatica, fu deciso di non modificare la terapia in corso e di rivalutare la densitometria ossea a un anno di distanza.

Nel luglio del 2016, il dato densitometrico mostrò un peggioramento ( $\mathrm{T}$ score $=-4.3$ ). Dal momento che la paziente aveva completato l'iter chirurgico odontoiatrico, fu ripristinata una terapia antiriassorbitiva, prescrivendo Denosumab $60 \mathrm{mg}$ (1 flacone ogni 6 mesi).

II controllo ecotomografico renale del 2016 dimostrò un quadro sostanzialmente immodificato rispetto al 2006, 
costituito da un calcolo di 6-7 $\mathrm{mm}$ a livello dei calici superiori di sinistra e un calcolo mesorenale destro di 4-5 mm.

\section{Conclusioni}

La normalizzazione dell'apporto nutrizionale di calcio e vitamina $D$ è una procedura raccomandata nel trattamento dell'Osteoporosi (8). La compresenza di litiasi renale non deve costituire un ostacolo al perseguimento di questo obiettivo. Oltretutto, una restrizione dell'apporto alimentare di calcio rispetto alle quantità raccomandate può addirittura avere effetti sfavorevoli sul rischio litogeno urinario (7).

Non è agevole ottenere regimi alimentari che forniscano adeguate quantità di calcio senza l'impiego di latte e derivati. Peraltro, a causa della quantità di sale, calorie e lipidi in essi contenuta, l'assunzione quotidiana di questi alimenti è sconsigliata nei soggetti ipertesi, dislipidemici o in sovrappeso corporeo.

Le acque bicarbonato-calciche costituiscono una valida fonte nutrizionale di calcio, altamente biodisponibile, in grado di sostituire la quota parte dietetica abitualmente coperta dal latte e dai suoi derivati. Nei soggetti con nefrolitiasi, esse sono particolarmente indicate per normalizzare l'apporto calcico, in quanto, rispetto ai derivati del latte, esse non contengono fattori calciuretici (proteine animali, sale) e hanno un effetto alcalinizzante, che aumenta l'escrezione urinaria di citrato $(11,13)$ e favorisce bilanci calcici positivi (14).

\section{Caso clinico 2}

\section{Anamnesi}

Paziente nata nel 1948. Menopausa a 53 anni. Sostanziale benessere fino all'età di 55 anni, quando furono riscontrate ipertensione arteriosa e dislipidemia e fu prescritta una terapia con Amlodipina e Simvastatina.

Nel maggio del 2012, a causa di lombalgie persistenti, fu eseguita un'ecografia che indicò la presenza di nefrolitiasi bilaterale, sino ad allora asintomatica. Una successiva TAC addominale dimostrò la presenza di un calcolo a stampo di $2 \mathrm{~cm}$ al polo inferiore del rene destro e uno di $7 \mathrm{~mm}$ al polo inferiore del rene sinistro, in assenza di una dilatazione delle vie escretrici, con vescica regolare.

Furono eseguiti anche alcuni esami ematochimici di base (emocromo, glicemia, uricemia, creatininemia, sodiemia, potassiemia, profilo epatico, esame completo delle urine) risultati nella norma, con i quali la paziente venne inviata a visita nefrologica.

\section{Indagini biochimiche e strumentali}

Nella Tabella II sono riportati gli esiti delle indagini metaboliche salienti eseguite dalla paziente durante il follow-up nefrologico.

Nel corso delle prime indagini (Basale), risultarono valori sopranormali di calcemia totale e ionizzata, una moderata ipofosforemia ed elevati livelli di PTH intatto circolante.

TABELLA II - Caso clinico 2. Dati metabolici

\begin{tabular}{|c|c|c|c|c|}
\hline & Val. normali & Basale & Cinacalcet & PTX \\
\hline Creatininemia (mg/dL) & $0.5-1.3$ & 0.8 & 0.9 & 1.0 \\
\hline Clear. Creatinina $(\mathrm{mL} / \mathrm{min})$ & $70-140$ & 90 & 88 & 84 \\
\hline Calcemia totale $(\mathrm{mg} / \mathrm{dL})$ & $8.2-10.2$ & 11.5 & 10.6 & 9.2 \\
\hline $\mathrm{Ca}^{++}$sierico $(\mathrm{mmol} / \mathrm{L})$ & $1.15-1.30$ & 1.49 & 1.36 & 1.15 \\
\hline Fosforemia $(\mathrm{mg} / \mathrm{dL}$ ) & $2.5-4.5$ & 1.9 & 2.3 & 3.3 \\
\hline PTH intatto $(p g / m L)$ & $11-70$ & 235 & 189 & 62 \\
\hline Fosfaturia (mmol/24h) & $13-52$ & 29.6 & 30.6 & 20.5 \\
\hline Calciuria ( $\mathrm{mmol} / 24 \mathrm{~h})$ & $2.5-9$ & 12.5 & 11.6 & 5.5 \\
\hline Calciuria (mg/kg) & $<4$ & 7.9 & 7.3 & 3.5 \\
\hline Ossaluria (mmol/24h) & $<0.55$ & 0.22 & 0.31 & 0.32 \\
\hline Citraturia (mmol/24h) & $1.5-6.5$ & 2.9 & 2.6 & 2.3 \\
\hline $\mathrm{pH}$ urinario & $5-7$ & 5.32 & 5.67 & 5.76 \\
\hline Diuresi (mL/24h) & & 2300 & 2400 & 3300 \\
\hline B CaOx & Satur. $=1$ & 5.93 & 6.1 & 2.7 \\
\hline B bsh & Satur. $=1$ & 0.85 & 0.88 & 0.7 \\
\hline
\end{tabular}


L'osteodensitometria lombare indicò una modesta osteopenia (T score $=-1.9$ ).

\section{Diagnosi}

Fu posta diagnosi di nefrolitiasi bilaterale in Iperparatiroidismo primitivo (PHPT).

La documentazione morfologica non è indispensabile per questa diagnosi (16); tuttavia, il riscontro topografico di un adenoma paratiroideo (la più frequente causa di PHPT) è, comunque, utile per consentire al chirurgo di eseguire un intervento mini-invasivo.

Furono, dunque, prescritte un'ecografia della loggia tiroidea e una scintigrafia paratiroidea con Tc99m Sestamibi. Entrambe non furono in grado di evidenziare con certezza la presenza di tessuto paratiroideo iperplastico o iperfunzionante.

\section{Terapia}

La paratiroidectomia è tuttora il trattamento di elezione del PHPT sintomatico (15), ma la paziente rifiutò la proposta di intervento chirurgico. Nel tentativo di ottenere comunque il controllo della sindrome ipercalcemica, fu prescritto cinacalcet $30 \mathrm{mg} \times 2$, programmando una rivalutazione biochimica a distanza di 4 mesi $(17,18)$.

\section{Decorso clinico}

In corso di terapia con calciomimetici, i valori di PTH e calcemia diminuirono, pur senza rientrare nei range di normalità (Tab. II), secondo un andamento ripetutamente riscontrato in Letteratura $(19,20)$.

La biochimica urinaria, in particolare la calciuria, non mostrò rilevanti variazioni durante la terapia e la sovrasaturazione per i sali litogeni rimase sostanzialmente immodificata, come da noi più volte riscontrato in casi analoghi (21).

Il cinacalcet fu poco tollerato dalla paziente, che accusò episodi di nausea e vomito, che la indussero ad accettare l'intervento di paratiroidectomia.

Fu effettuata una cervicotomia esplorativa (le indagini morfologiche non avevano, infatti, evidenziato tessuto iperplastico), nel corso della quale fu rinvenuto e asportato un adenoma a carico della paratiroide inferiore sinistra. La completezza dell'intervento fu confermata dal dosaggio intraoperatorio del PTH, che, al termine dell'exeresi dell'adenoma, risultò in limiti di norma (22 pg/mL vs 161 pg/mL pre-exeresi).

Una rivalutazione metabolica effettuata a 6 mesi di distanza confermò una condizione di euparatiroidismo, con normalizzazione dei livelli circolanti di calcio, fosforo e PTH e con la completa regressione dell'ipercalciuria e il conseguente miglioramento della sovrasaturazione urinaria rispetto ai sali di calcio (Tab. II, PTX).

\section{Conclusioni}

II PHPT è in grado di indurre alterazioni della biochimica urinaria favorenti la litogenesi calcica, in particolare un'aumentata escrezione di calcio e fosforo $(22,23)$. Altri fattori, in modo variabile, possono cooperare ad aumentare la saturazione urinaria per i sali di calcio in pazienti con PHPT, come una propensione all'alcalinizzazione urinaria (24) e a una lieve iperossaluria da aumentato assorbimento intestinale di ossalato (in questo caso, non evidenziabile). Quest'ultimo fenomeno può essere favorito dalle peculiari modificazioni dell'assorbimento intestinale del calcio indotte dal calcitriolo (25), la cui sintesi metabolica è aumentata in corso di PHPT.

Benché il cinacalcet possa consentire di correggere l'ipercalcemia nei soggetti iperparatiroidei, esso non è parimenti efficace né nel controllare la sindrome ipercalciurica, verosimilmente a causa dei suoi effetti diretti a livello del tubulo renale (26), né nel correggere la fosfaturia indotta dal PTH, i cui livelli tendono a rimanere superiori alla norma anche in corso di terapia con i calciomimetici $(19,20)$.

Il caso clinico qui descritto conferma come, nei pazienti con PHPT complicato da nefrolitiasi, la terapia con calciomimetici non possa essere considerata un sostituto della paratiroidectomia, che resta, a oggi, l'unica terapia in grado di ripristinare una vera condizione di euparatiroidismo e di normalizzare il rischio litogeno urinario (21).

\section{Disclosures}

Financial support: No financial support was received for this submission. Conflict of interest: The authors have no conflict of interest.

\section{Bibliografia}

1. Ramello A, Vitale C, Marangella M. Epidemiology of nephrolithiasis. J Nephrol. 2000;13(Suppl. 3):S45-50.

2. Trinchieri A, Coppi $F$, Montanari $E$, et al. Increase in the prevalence of symptomatic upper urinary tract stones during the last ten years. Eur Urol. 2000;37:23-5.

3. Croppi E, Ferraro PM, Taddei L, Gambaro G. Prevalence of renal stones in an Italian urban population: a general practice-based study. Urol Res. 2012;40(5):517-22.

4. Pak CY, Poindexter JR, Adams-Huet B, Pearle MS. Predictive value of kidney stone composition in the detection of metabolic abnormalities. Am J Med. 2003;115:26-32.

5. Marangella M, Daniele PG, Ronzani M, Sonego S, Linari F. Urine saturation with calcium salts in normal subjects and idiopathic calcium stone formers estimated by an improved computer model system. Urol Res. 1985;13:189-93.

6. Rejnmark L, Vestergaard P, Mosekilde L. Nephrolithiasis and Renal Calcifications in Primary Hyperparathyroidism. J Clin Endocrinol Metab. 2011;96:2377-85.

7. Curhan GC, Willett WC, Rimm EB, Stampfer MJ. A prospective study of dietary calcium and other nutrients and the risk of symptomatic kidney stones. N Engl J Med. 1993;328(12):833-8.

8. Società Italiana dell'Osteoporosi, del Metabolismo Minerale e delle Malattie dello Scheletro. Linee Guida per la diagnosi, la prevenzione e la terapia dell'osteoporosi. Disponibile su http:// www.siommms.it/category/linee-guida/ (Accesso: 14/10/2016). 
9. Curhan GC, Willett WC, Speizer FE, et al. Comparison of dietary calcium with supplemental calcium and other nutrients as factors affecting the risk for kidney stones in women. Ann Intern Med. 1997;126:497-504.

10. Elenco acque minerali. Disponibile su http://www.acqueminerali.it (accesso 14/10/2016).

11. Marangella $M$, Vitale $C$, Petrarulo $M$, Rovera L, Dutto F. Effects of mineral composition of drinking water on risk for stone formation and bone metabolism in idiopathic calcium nephrolithiasis. Clin Sci. 1996;91(3):313-8.

12. Vitale $C$, Marangella $M$, Manganaro $M$, Petrarulo $M$, Rovera $L$, Ramello A. Effetti metabolici di un'acqua minerale bicarbonato calcica nella terapia dietetica dell'insufficienza renale cronica. G Ital Nefrol. 1999;16(2):144-7.

13. Simpson DP. Citrate excretion: a window on renal metabolism. Am J Physiol. 1983;244:F223-34.

14. Sebastian A, Harris ST, Ottaway JH, Todd KM, Morris RC Jr. Improved Mineral Balance and Skeletal Metabolism in Postmenopausal Women Treated with Potassium Bicarbonate. N Engl J Med. 1994;330:1776-81.

15. Marangella M, Bianco O, Martini C, Petrarulo M, Vitale C, Linari $\mathrm{F}$. Effect of animal and vegetable protein intake on oxalate excretion in idiopathic calcium stone disease. Br J Urol. 1989;63(4):348-51.

16. The American Association of Clinical Endocrinologists and the American Association of Endocrine Surgeons position statement on the diagnosis and management of primary hyperparathyroidism. Endocrine Practice. 2005;11(1):49-54.

17. Marcocci C, Chanson P, Shoback D, et al. Cinacalcet reduces serum calcium concentrations in patients with intractable primary hyperparathyroidism. J Clin Endocrinol Metab. 2009;94:2766-72.
18. Schwarz P, Body JJ, Cap J, et al. The PRIMARA study: a prospective, descriptive, observational study to review cinacalcet use in patients with primary hyperparathyroidism in clinical practice. Eur J Endocrinol. 2014;171:727-35.

19. Iglesias P, Ais G, González A, et al. Acute and one-year effects of cinacalcet in patients with persistent primary hyperparathyroidism after unsuccessful parathyroidectomy. Am J Med Sci. 2008;335(2):111-4.

20. Luque-Fernández I, García-Martín A, Luque-Pazos A. Experience with cinacalcet in primary hyperparathyroidism: results after 1 year of treatment. Ther Adv Endocrinol Metab. 2013;4(3):77-81.

21. Vitale C, Bermond F, Rodofili A, et al. Effetti del Cinacalcet sulla biochimica urinaria e sul rischio litogeno in pazienti con Iperparatiroidismo Primitivo e calcolosi renale. G Ital Nefrol. 2016;33(4):Pii:gin/33.4.7.

22. Odvina CV, Sakhaee K, Heller HJ, et al. Biochemical characterization of primary hyperparathyroidism with and without kidney stones. Urol Res. 2007;35(3):123-8.

23. Starup-Linde J, Waldhauer E, Rolighed L, Mosekilde L, Vestergaard P. Renal stones and calcifications in patients with primary hyperparathyroidism: associations with biochemical variables. Eur J Endocrinol. 2012;166:1093-100.

24. Fan L, Wiederkehr MR, Collazo R, Wang H, Crowder LA, Moe $\mathrm{OW}$. Dual mechanisms of regulation of $\mathrm{Na} / \mathrm{H}$ exchanger NHE-3 by parathyroid hormone in rat kidney. J Biol Chem. 1999;274(16):11289-95.

25. Marangella $M$, Vitale $C$, Cosseddu D, Petrarulo $M$, Linari F. Effects of oral and intravenous calcitriol on serum calcium oxalate saturation in dialisi patients. Clin Sci. 1993;85(3):309-14.

26. Riccardi D, Brown EM. Physiology and pathophysiology of the calcium sensing receptor in the kidney. Am J Physiol Renal Physiol. 2009;298:F485-99. 